Cerium oxide nanoparticles coated by surfactant sodium bis(2-ethylhexyl) sulphosuccinate (AOT): local atomic structures and $x$-ray absorption spectroscopic studies

This content has been downloaded from IOPscience. Please scroll down to see the full text.

2001 J. Phys.: Condens. Matter 135269

(http://iopscience.iop.org/0953-8984/13/22/320)

View the table of contents for this issue, or go to the journal homepage for more

Download details:

IP Address: 134.58.253.57

This content was downloaded on 28/04/2014 at 18:57

Please note that terms and conditions apply. 


\title{
Cerium oxide nanoparticles coated by surfactant sodium bis(2-ethylhexyl) sulphosuccinate (AOT): local atomic structures and x-ray absorption spectroscopic studies
}

\author{
Zhonghua $\mathrm{Wu}^{1,2,4}$, Robert E Benfield ${ }^{2}$ Lin Guo $^{1}$ Huanjun $\mathbf{L i}^{3}$,

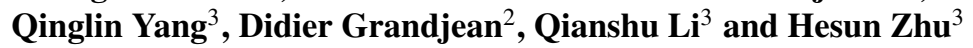 \\ ${ }^{1}$ BSRF, Institute of High Energy Physics, Chinese Academy of Sciences, PO Box 918, Bin 2-7, \\ Beijing 100039, People's Republic of China \\ 2 Centre for Materials Research, School of Physical Sciences, University of Kent, \\ Canterbury CT2 7NR, UK \\ ${ }^{3}$ College of Chemical Engineering and Material Science, Beijing Institute of Technology, \\ PO Box 327, Beijing 100081, People's Republic of China \\ E-mail:wuzh@mail.ihep.ac.cn and z.wu@ukc.ac.uk
}

Received 1 February 2001, in final form 26 March 2001

\begin{abstract}
Cerium oxide nanoparticles coated by sodium bis(2-ethylhexyl) sulphosuccinate (AOT) were prepared by using a microemulsion method. Transmission electron microscopy revealed an average particle size of 2-3 nm. X-ray diffraction showed that the cerium oxide nanoparticles retain the $\mathrm{CeF}_{2}$-type cubic structures like the bulk crystal. The intermediate valence of formally tetravalent compounds had been detected by $\mathrm{x}$-ray-absorption near-edge struc-

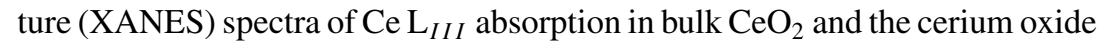
nanoparticles. Two well resolved white lines can be assigned to the electron configurations of $4 \mathrm{f}^{0} \mathrm{~L}$ and $4 \mathrm{f}^{1} \mathrm{~L}$, respectively, where $\mathrm{L}$ denotes a ligand hole. At the same time, the cerium oxide nanoparticles also showed the structural features of trivalent compounds, in comparison to the trivalent $\mathrm{Ce}\left(\mathrm{NO}_{3}\right)_{3} \cdot 6 \mathrm{H}_{2} \mathrm{O}$. Four Lorentzian functions and two arctan functions were used to fit the normalized XANES spectra. The extended x-ray-absorption fine-structure (EXAFS) technique was used to probe the local atomic structures around the absorber Ce. The multielectron excitation effect on the EXAFS spectra was eliminated. A core-shell model was used to deduce the near-neighbour structural parameters around cerium. Bulk $\mathrm{CeO}_{2}$ with eight oxygen atoms located at $2.343 \AA$ was used as the reference sample to extract the backscattering amplitude and phase shift of the $\mathrm{Ce}-\mathrm{O}$ bond. One half of the atoms locate at the core part with the $\mathrm{CeF}_{2}$-type cubic structures (eight oxygens at $2.343 \AA$ around $\mathrm{Ce}$ ), the other half of the atoms are amorphous phase located in the shell part (surface of the nanoparticles) with approximately $\mathrm{Ce}_{2} \mathrm{O}_{3}$ structural features (averaged seven oxygens at $2.50 \AA$ around Ce).
\end{abstract}

\footnotetext{
4 To whom correspondence should be addressed.
} 


\section{Introduction}

Because of the high surface to volume ratio and quantum-size effect [1,2], nanometre scale materials present unusual chemical and physical properties different from those of bulk materials. Recently, there has been great scientific interest [3-12] in synthesis of $\mathrm{CeO}_{2}$ nanoparticles and modifications of their size, morphology and property for a large variety of applications such as ultraviolet absorbent and glass-polishing materials and three-way catalysts for the automotive industry.

For cerium oxide nanoparticles, interest focuses on not only their atomic structures, but also their electron structures. The covalent mixing [13-16] of $4 \mathrm{f}$ states and multielectron resonance [17-21] at the $\mathrm{Ce}_{I I I}$ edge make the $\mathrm{x}$-ray absorption spectroscopy more complicated. The $\mathrm{x}$-ray-absorption near-edge structure (XANES) spectroscopy of the $\mathrm{Ce}_{I I I}$ edge is full of information on electron structures such as the final-state mixed-valence behaviour, the intense multielectron excitations and chemical valence states. Some spectroscopic features are closely connected with atomic and electronic structures. At the same time, they also bring difficulties in the extended x-ray-absorption fine-structure (EXAFS) data analysis. Studying the x-ray absorption spectroscopy of the $\mathrm{Ce} \mathrm{L}_{I I I}$ edge has not only practical application, but also theoretical importance.

X-ray absorption spectroscopy has revealed itself as a powerful tool for structural characterization of nanometre scale materials because of its element selectivity and independence of the long-range order of materials. Much theoretical and experimental research has been reported on the final-state mixed valence behaviour and the intense multielectron excitations of cerium oxides and compounds at the $\mathrm{L}_{I I I}$ edge of cerium, but few reports $[18,22]$ can be found for their EXAFS studies. The synthesis of the nano-scaled cerium oxide nanoparticles promotes us to examine and compare their local atomic structures and the spectroscopic features with the bulk counterpart.

In this paper, cerium oxide nanoparticles coated with sodium bis(2-ethylhexyl) sulphosuccinate (AOT) were prepared. By using the $\mathrm{x}$-ray-absorption spectroscopy technique, we found a large change in the XANES spectrum of the $\mathrm{Ce}_{I I I}$ edge in comparison with the bulk one. The local atomic structures around $\mathrm{Ce}$ in cerium oxide nanoparticles were also probed. We expect that this is helpful for explaining the peculiar properties of these nanoparticles.

\section{Experiment}

\subsection{Sample preparation}

Cerium oxide nanoparticles had been prepared with different methods [3-12]. Recently, the liquid-liquid two-phase system [12] has been proven to be a useful technique for preparing novel materials with unusual properties. In this paper, we have modified the method and employed directly surfactant (AOT) as both protective agent and phase-transfer reagent to prepare cerium oxide nanoparticles. All the chemicals and reagents used were of analytical grade. AOT, cerium nitrate hexahydrate, toluene and ammonium hydroxide were commercially available. The synthesis process was described as follows:

$5 \mathrm{ml}$ of $0.1 \mathrm{~mol} \mathrm{l}^{-1}$ cerium nitrate aqueous solution was added to a solution of $1.5 \mathrm{~g}$ of AOT surfactant in $100 \mathrm{ml}$ toluene under vigorous stirring, and the mixture solution was stirred rapidly for $0.5 \mathrm{~h}$. Then continuing to stir, $10 \mathrm{ml}$ of $1.5 \mathrm{~mol}^{-1}$ ammonium hydroxide aqueous solution was slowly added dropwise; the solution gradually turned bright yellow, indicating the formation of colloidal particles. After that the reaction mixture was further stirred for $1 \mathrm{~h}$ and 
the solution colour gradually became deep. Finally this mixture was left to stand until it was thoroughly separated into two layers. The upper layer was the toluene sol containing yellow nanoparticles, and the lower layer was aqueous phase. The organic phase was collected. The nanometre cerium oxide powder was obtained by removing the solvent from the organic sol.

\subsection{TEM and XRD characterization}

The cerium oxide nanoparticles were characterized using transmission electron microscopy (JEM 200-CX) with an accelerating voltage of $100 \mathrm{kV}$. The TEM photograph of the sample is presented as figure 1 . The particle size can be found to distribute in a range of 2-3 nm.

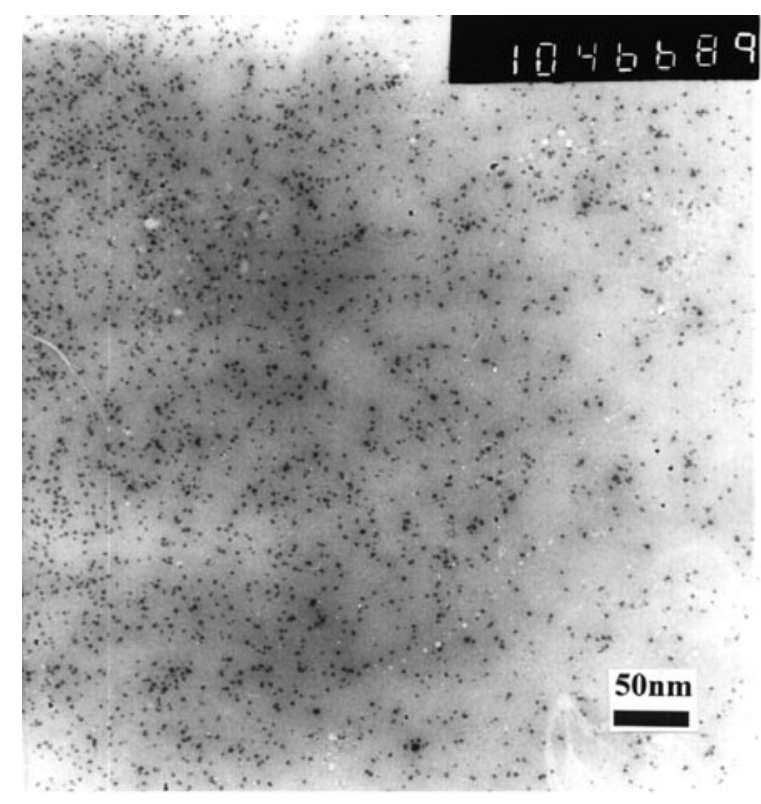

Figure 1. Transmission electron micrograph of AOT-coated cerium oxide nanoparticles.

Figure 2 shows the x-ray diffraction (XRD) patterns of bulk $\mathrm{CeO}_{2}$ and cerium oxide nanoparticles, which were recorded on a Rigaku RINT/Dmax-2000 diffractometer using $\mathrm{Cu} \mathrm{K} \alpha$ radiation. The nanoparticles present a set of broadened diffraction peaks that reflect the very small particle size. Obviously, the diffraction peaks of cerium oxide nanoparticles correspond one to one with those of bulk $\mathrm{CeO}_{2}$ and can be roughly indexed as (111), (200), (220) and (311) allowed reflecting planes expected from the cubic fluorite structure $\mathrm{CeO}_{2}$. For comparing and identifying the crystalline phase of nanostructural cerium oxides, the relative peak position and intensity of $\mathrm{Ce}_{2} \mathrm{O}_{3}$ is also drawn in figure 2. We cannot find identifiable diffraction peaks of crystalline $\mathrm{Ce}_{2} \mathrm{O}_{3}$ phase from the XRD pattern of these nanoparticles. From the TEM photograph and the XRD pattern, we think that these cerium oxide nanoparticles are approximately the same size and kind.

\subsection{Collection of $x$-ray absorption spectra}

X-ray absorption spectra were collected on the EXAFS station (beam line 4W1B) of Beijing Synchrotron Radiation Facility. The cerium oxide nanoparticles were homogeneously smeared 


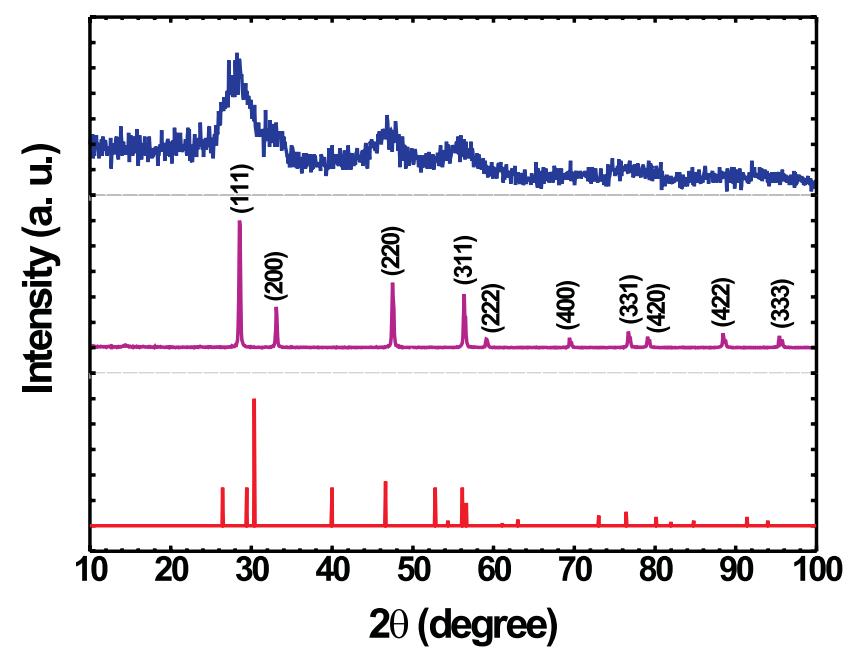

Figure 2. X-ray diffraction patterns of AOT-coated cerium oxide nanoparticles (top), bulk $\mathrm{CeO}_{2}$ (middle) and bulk $\mathrm{Ce}_{2} \mathrm{O}_{3}$ (botom; only the intensity and position).

on Scotch adhesive tape. More than eight layers were folded to reach the optimum absorption thickness $(\Delta \mu d \approx 1.0 ; \Delta \mu$ is the absorption edge step, $d$ is the physical thickness of the sample). X-ray absorption spectra of the $\mathrm{Ce}_{\text {III }}$ edge of AOT-coated cerium oxide nanoparticles and bulk $\mathrm{CeO}_{2}$ were collected at ambient temperature in transmission mode. Three independent scans were carried out for each spectrum. The storage ring was run at $2.2 \mathrm{GeV}$ with the electron current about $50 \mathrm{~mA}$. Higher harmonics were eliminated by detuning the double crystal $\mathrm{Si}(111)$ monochromator. The incident and transmission $\mathrm{x}$-ray intensities were, respectively, detected by ion chambers that were installed in front of and behind the sample. X-ray energy was calibrated by using the $\mathrm{Cr} \mathrm{K}$ absorption edge (5989 eV). Energy resolution $(\Delta E / E)$ is about $2 \times 10^{-4}$. The absorption spectra were collected from $200 \mathrm{eV}$ below the absorption threshold to over $500 \mathrm{eV}$ above the threshold until the $\mathrm{L}_{I I}$ absorption edge appeared. The normalized absorption curves are shown in figure 3.

\section{XANES spectroscopy}

Figure 4 shows the Ce- $\mathrm{L}_{I I I}$ XANES spectra of bulk $\mathrm{CeO}_{2}$, cerium oxide nanoparticles and hydrous cerium nitrate $\left(\mathrm{Ce}\left(\mathrm{NO}_{3}\right)_{3} \cdot 6 \mathrm{H}_{2} \mathrm{O}\right)$. The energy corresponding to the maximum of the first derivative of the absorption edge, was chosen as the energy threshold. Four features can be found from the XANES spectra, the high-energy feature A, the main peak B, the lowenergy feature C and the pre-edge peak D. Peaks A and B were described as mixing of the multielectron configurations [14]: $4 \mathrm{f}^{0} \mathrm{~L}$ and $4 \mathrm{f}^{1} \mathrm{~L}$, where $\mathrm{L}$ denotes a ligand $(\mathrm{O} 2 \mathrm{p})$ hole. The feature $A$ was also separated into two subpeaks $A_{1}$ and $A_{2}$ corresponding to the crystal field split of the $4 \mathrm{f}^{0} \mathrm{~L}$ final state [22]. The low-energy shoulder $\mathrm{C}$ was due to the crystal field splitting of $\mathrm{Ce} 5 \mathrm{~d}$ states for bulk $\mathrm{CeO}_{2}$ [14] or was assigned to a $\mathrm{Ce}^{3+}$ impurity [16, 23]. The pre-edge peak $\mathrm{D}$ was the result [14] of the transition to the unoccupied $\mathrm{Ce} d$ states at the bottom of $\mathrm{CeO}_{2}$ conduction band or a dipole-forbidden $2 \mathrm{p}_{3 / 2} \rightarrow 4 \mathrm{f}$ transition [22], which is a consequence of $5 \mathrm{~d}$ admixtures to the $4 \mathrm{f}$ state. However, for cerium oxide nanoparticles in this paper, the feature $\mathrm{B}$ is merged into the feature $\mathrm{C}$ and feature $\mathrm{D}$ disappears. The feature $\mathrm{C}$ is drastically increased in comparison with one of the bulk $\mathrm{CeO}_{2}$. 


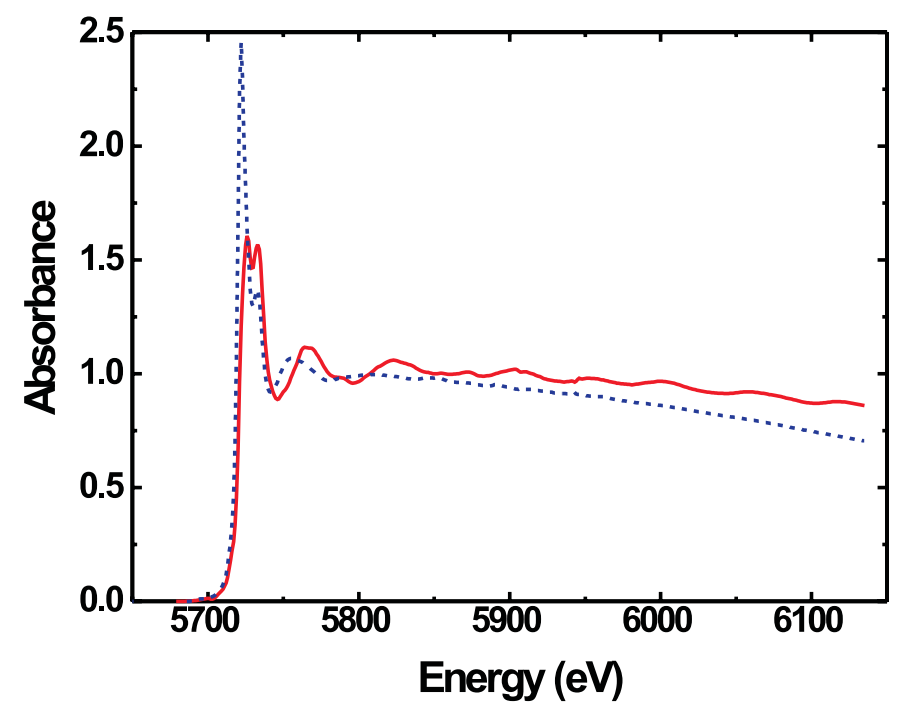

Figure 3. Normalized $\mathrm{x}$-ray absorption curves of $\mathrm{L}_{I I I}$ edge in cerium oxide nanoparticles (dashed line) and bulk $\mathrm{CeO}_{2}$ (solid line)

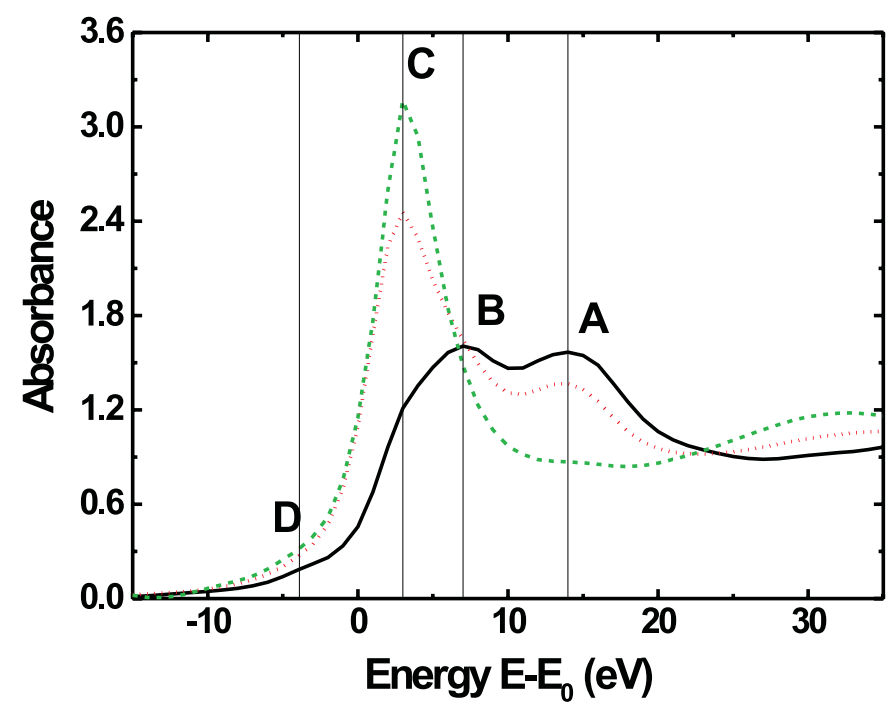

Figure 4. X-ray near-edge absorption spectra of AOT-coated cerium oxide nanoparticles (dotted line), bulk $\mathrm{CeO}_{2}$ (solid line) and hydrous cerium nitrate $\left(\mathrm{Ce}\left(\mathrm{NO}_{3}\right)_{3} \cdot 6 \mathrm{H}_{2} \mathrm{O}\right)$ (dashed line). Four features A, B, C and D are denoted by the vertical fine lines.

Feature $\mathrm{A}$ and $\mathrm{B}$ are characteristic of $\mathrm{Ce}^{4+}$ oxides and compounds. However the $\mathrm{Ce}^{3+}$ compounds, for examples $\mathrm{CeF}_{3}$ [24] and $\mathrm{Ce}\left(\mathrm{NO}_{3}\right)_{3} \cdot 6 \mathrm{H}_{2} \mathrm{O}$ herein, have obviously different spectral features. The main peak of $\mathrm{Ce}\left(\mathrm{NO}_{3}\right)_{3} \cdot 6 \mathrm{H}_{2} \mathrm{O}$ has quite high intensity and coincides with the low-energy shoulder $\mathrm{C}$ of bulk $\mathrm{CeO}_{2}$. This spectral difference is an important characteristic of distinguishing $\mathrm{Ce}^{3+}$ and $\mathrm{Ce}^{4+}$ compounds. From figure 4 , it can been seen that the shape and energy position of peak $\mathrm{C}$ of cerium oxide nanoparticles are the same as those of the main peak of $\mathrm{Ce}\left(\mathrm{NO}_{3}\right)_{3} \cdot 6 \mathrm{H}_{2} \mathrm{O}$. This implies that these nanoparticles present partially the characteristics of 
three-valent $\mathrm{Ce}^{3+}$. However, an obvious feature $\mathrm{A}$ for the cerium oxide nanoparticle spectrum is exactly the contribution of $\mathrm{Ce}^{4+}$. This phenomenon leads us to consider a possible mixed phase of $\mathrm{Ce}^{3+}$ and $\mathrm{Ce}^{4+}$. Evidently, the crystalline $\mathrm{Ce}^{4+}$ phase is inevitable as indicated by the XRD patterns. It implies an amorphous $\mathrm{Ce}^{3+}$ phase possibly existing. This is confirmed by the EXAFS results (see below).

The XANES spectrum of cerium oxide nanoparticles cannot be simulated well by combining the two components of bulk $\mathrm{CeO}_{2}$ and $\mathrm{Ce}\left(\mathrm{NO}_{3}\right)_{3} \cdot 6 \mathrm{H}_{2} \mathrm{O}$ as shown in figure 5 . This demonstrates that the local atomic structures and symmetry of the surface area of the nanoparticles are not the same as those of $\mathrm{Ce}\left(\mathrm{NO}_{3}\right)_{3} \cdot 6 \mathrm{H}_{2} \mathrm{O}$, although they both have the same valence of $\mathrm{Ce}^{3+}$. However, the XANES spectrum of the $\mathrm{Ce}_{I I I}$ edge of the nanoparticles can still be separated into two components, i.e. the $\mathrm{Ce}^{4+}$ component $(51 \%)$ of bulk- $\mathrm{CeO}_{2}$ and the $\mathrm{Ce}^{3+}$ component $(49 \%)$ of probably $\mathrm{Ce}_{2} \mathrm{O}_{3}$. The $\mathrm{Ce}^{3+}$ component has the similar shape as that of the $\mathrm{Ce}\left(\mathrm{NO}_{3}\right)_{3} \cdot 6 \mathrm{H}_{2} \mathrm{O}$, but higher intensity.

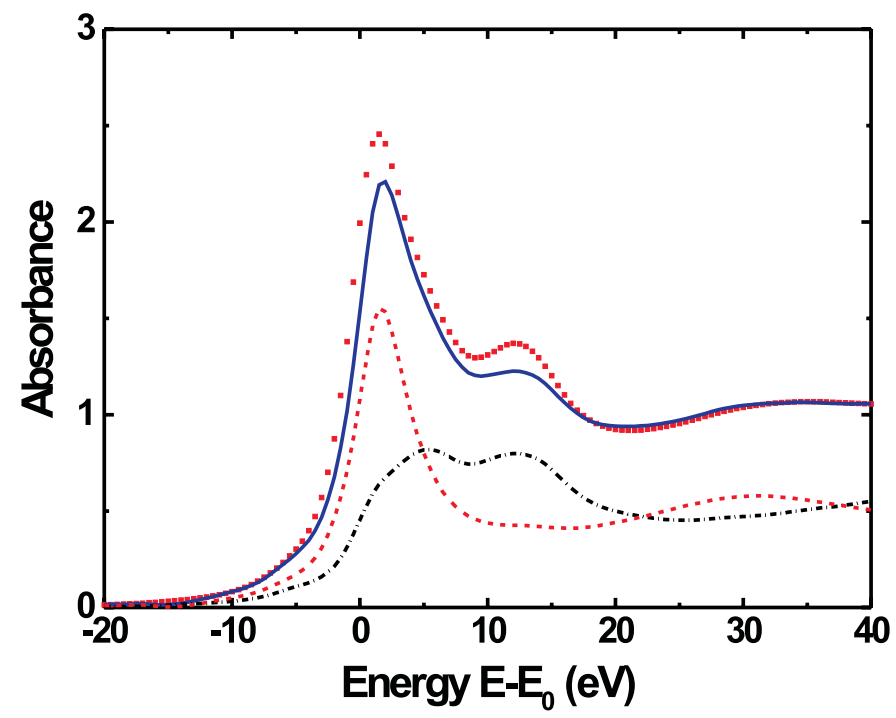

Figure 5. Experimental XANES spectrum (dotted line) and its simulated curve (solid line) by combining the two components of bulk $\mathrm{CeO}_{2}\left(51 \%\right.$, dot-dashed line) and $\mathrm{Ce}\left(\mathrm{NO}_{3}\right)_{3} \cdot 6 \mathrm{H}_{2} \mathrm{O}(49 \%$, the dashed line) for the $\mathrm{Ce} \mathrm{L}_{I I}$ edge of cerium oxide nanoparticles. Clearly, the experimental XANES spectrum cannot be simulated well by combining the two components.

For XANES analysis, a linear pre-edge background was fitted and removed. The edge height was fitted and normalized to unity with an arctangent function. The post-edge background was modified to the constant unity. The maximum of the first derivative of the edge region was chosen as the energy threshold. The XANES spectra of bulk $\mathrm{CeO}_{2}$ and cerium oxides nanoparticles have been fitted with four Lorentzians $[25,26]$ as shown in figures 6 and 7 , respectively. Two arctangent curves were used to fit the absorption step edge, representing transitions of $4 \mathrm{f}^{0}$ and $4 \mathrm{f}^{1}$ configurations to the continuum. The positions of peak A, B C and $\mathrm{D}$ were, respectively fixed at $12.7,5.8,1.6$ and $-5.1 \mathrm{eV}$ for bulk $\mathrm{CeO}_{2}$ and the related nanoparticles, corresponding to the maximum of these peaks. The fitting areas (intensity) of the four peaks were, respectively, denoted as $I_{A}, I_{B}, I_{C}$ and $I_{D}$. The fitting parameters are listed in table 1.

Peaks $A$ and $B$ correspond to the transitions to the $5 \mathrm{~d}$ states of cerium with $4 \mathrm{f}^{0}$ and $4 \mathrm{f}^{1}$ valence configurations. The cerium spectroscopic valence $v_{s}$ is defined as $v_{s}=$ 


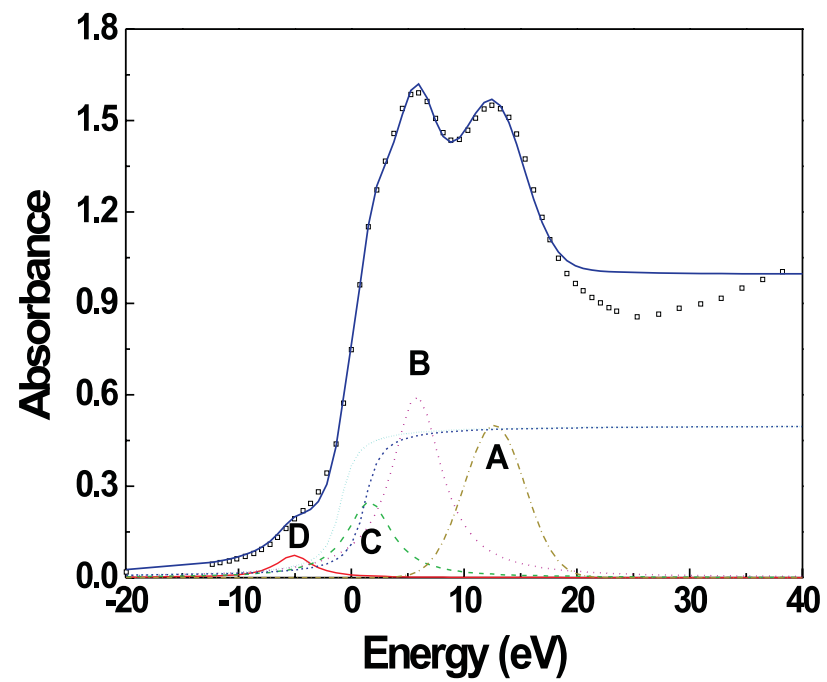

Figure 6. Fitting (solid line) of the experimental XANES spectrum (open squares) of the $\mathrm{Ce} \mathrm{L}_{I I I}$ edge of bulk $\mathrm{CeO}_{2}$ by using four resonance peaks A, B, C and D (respectively, dot-dash, dotted, dashed and solid lines), as well as two absorption backgrounds (short-dashed and short-dotted lines) corresponding to the configurations $4 \mathrm{f}^{0} \mathrm{~L}$ and $4 \mathrm{f}^{1} \mathrm{~L}$.

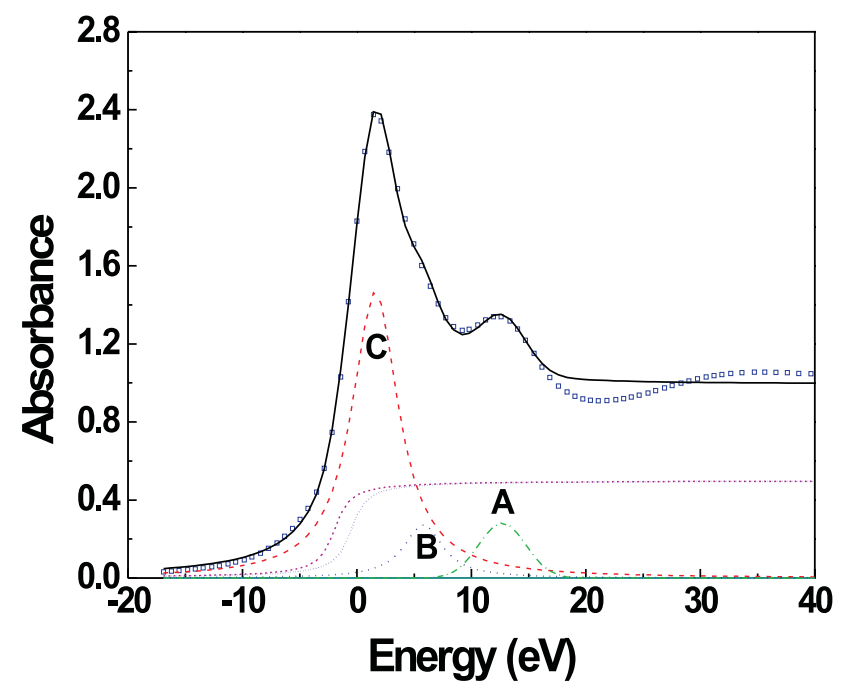

Figure 7. Fitting (solid line) of the experimental XANES spectrum (open squares) of the $\mathrm{Ce}_{I I I}$ edge of cerium oxide nanoparticles by using four resonance peaks A, B, C and D (respectively, dot-dash, dotted, dashed and solid lines), as well as two absorption backgrounds (short-dashed and short-dotted lines) corresponding to the configuration $4 \mathrm{f}^{0} \mathrm{~L}$ and $4 \mathrm{f}^{1} \mathrm{~L}$. The feature $\mathrm{D}$ almost disappears in this case.

$3+I_{A} /\left(I_{A}+I_{B}\right)$. The spectroscopic estimate of the f-electron count is then $n_{f}=\left(4-v_{s}\right)$. The spectroscopic valences are determined as 3.45 for bulk $\mathrm{CeO}_{2}$ and cerium oxide nanoparticles; the corresponding f-electron count is 0.55 . Similar results for bulk $\mathrm{CeO}_{2}$ can be found from previous reports $[16,23,27]$. For the cerium oxide nanoparticles, we should indicate that the same spectroscopic valence and f-electron counts were obtained as compared with the bulk- 
Table 1. Fitting parameters of the four characteristic peaks at the XANES spectra of the Ce $\mathrm{L}_{I I I}$ edge of bulk $\mathrm{CeO}_{2}$ and cerium oxide nanoparticles. Here, 'Width' means the half width at half height.

\begin{tabular}{|c|c|c|c|c|c|c|}
\hline \multirow[b]{2}{*}{ Peak } & \multicolumn{3}{|c|}{ Bulk $\mathrm{CeO}_{2}$} & \multicolumn{3}{|c|}{ Ce oxide nanoparticles } \\
\hline & Position (eV) & Width (eV) & Area $(\mathrm{eV})$ & Position (eV) & Width (eV) & Area $(\mathrm{eV})$ \\
\hline A & 12.7 & 2.8 & 3.53 & 12.7 & 2.0 & 1.43 \\
\hline B & 5.8 & 2.4 & 4.29 & 5.8 & 2.0 & 1.74 \\
\hline $\mathrm{C}$ & 1.6 & 2.9 & 2.73 & 1.6 & 2.5 & 11.45 \\
\hline $\mathrm{D}$ & -5.1 & 1.7 & 0.34 & -5.1 & 19.2 & 0.01 \\
\hline
\end{tabular}

$\mathrm{CeO}_{2}$ case based on the intensity (area) of the features A and B. Actually, this told us there is indeed a partial $\mathrm{CeO}_{2}$ component in the cerium oxide nanoparticles. This is consistent with the XRD results.

\section{Multielectron excitation}

It is well known that multielectron excitations contribute significantly to the near-edge structure in x-ray-absorption spectroscopy. However, multielectron features [28-30] can also exist at energies far above the edge. In EXAFS data analysis, the EXAFS oscillations are usually extracted assuming a smooth and monotonic background, which can be simulated by using a spline function or a polynomial. Obviously, a monotonic background cannot exclude the multielectron features, which will result in inappropriate results in the EXAFS region (in the range 40 to approximately $1000 \mathrm{eV}$ ). For $\mathrm{Ce}^{4+}$ compounds, the multielectron excitation at EXAFS region is inevitable. The theoretical calculations [21] demonstrate that the main double-excitation channel is the $2 \mathrm{p} 4 \mathrm{~d} \rightarrow(5 \mathrm{~d})^{2}$ transition, while the contributions from other channels are negligible. Fonda et al [18] compared the EXAFS spectra of bulk $\mathrm{CeO}_{2}$ with and without a shake-up resonance in the simulation, and found that a simple EXAFS simulation is unable to provide good results. Benfield et al [28] also indicated that double excitations which are not removed from EXAFS spectrum will cause large systematic error and hamper the location of the correct minimum. Therefore, the multielectron excitation should be subtracted

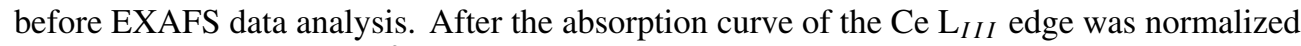
to unity, the $2 \mathrm{p} 4 \mathrm{~d} \rightarrow(5 \mathrm{~d})^{2}$ transition was simulated in energy space by using a Lorentzian curve [18] as follows:

$$
Y(E)=\frac{H}{1+\left(\left(E-E_{1}\right) / W\right)^{2}}
$$

where $E$ is the incident photon energy and $E_{1}$ is the position of the multielectron excitation. $H$ is the height of the peak and $W$ is the half width at half height. The heights were chosen as 0.034 as indicated by theoretical calculation [21]. Their position and width were adjusted until the unphysical peak in the low- $R$ region reached a minimum. The multielectron transitions were simulated in energy space as shown in figure 8 and 9, respectively, for the bulk $\mathrm{CeO}_{2}$ and the cerium oxide nanoparticles. Table 2 lists the parameters of these Lorentzian curves. It is about $5 \mathrm{eV}$ lower for the multielectron excitation position in the nanoparticles than in the bulk $\mathrm{CeO}_{2}$. This is caused by the addition of the $\mathrm{Ce}^{3+}$ component in the nanoparticles, which results in a red shift of the energy position. The width of the multielectron excitation is larger in the nanoparticles than in the bulk $\mathrm{CeO}_{2}$. This is probably caused by the larger structural disorder in the nanoparticles. 


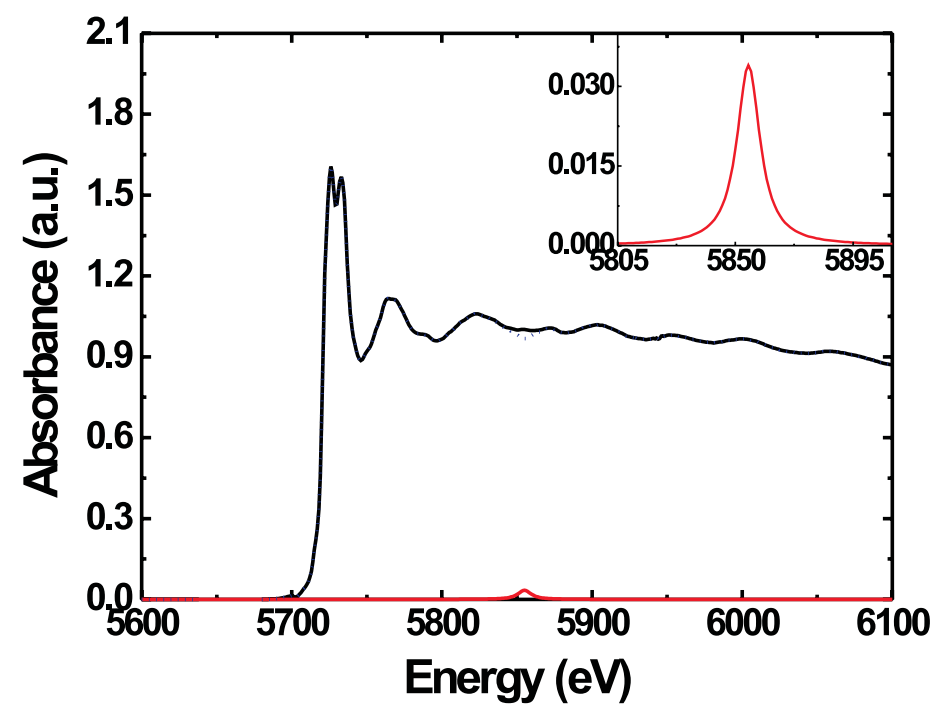

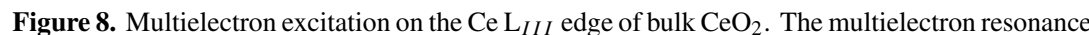
was fitted using a Lorentzian function (bottom) and is also shown in the inset; the raw absorption curve (solid line) and the one (dotted line) with removal of the multielectron resonance are also shown in this figure.

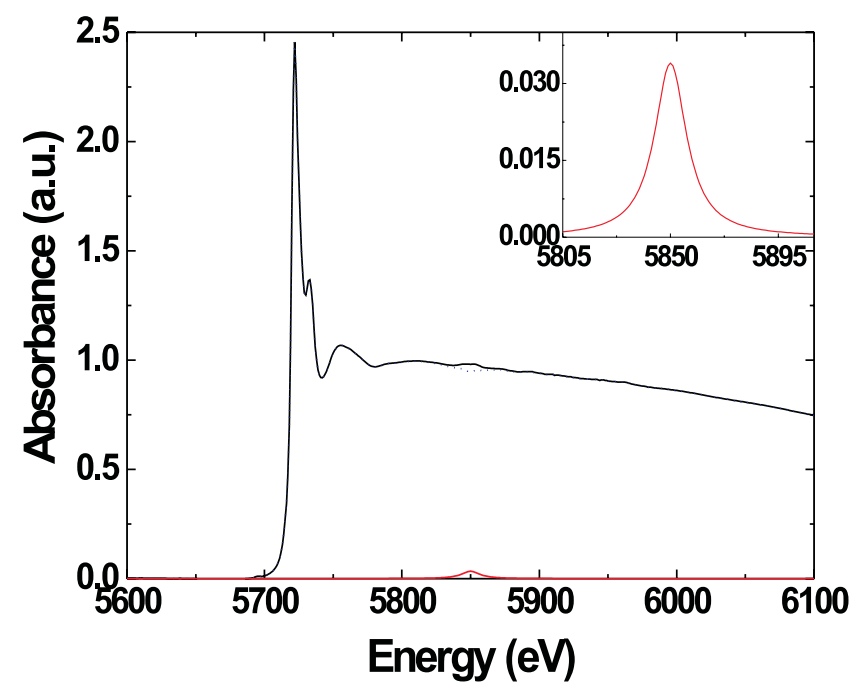

Figure 9. Multielectron excitation on the $\mathrm{Ce} \mathrm{L}_{I I I}$ edge of cerium oxide nanoparticles. The multielectron resonance was fitted using a Lorentzian function (bottom) and is also shown in the inset; the raw absorption curve (solid line) and the one (dotted line) with removal of the multielectron resonance are also shown in this figure.

\section{EXAFS data analysis}

After the removal of the multielectron excitation effect, the absorption curves were transferred to $k$ space using the formula $k=\left(2 m\left(E-E_{0}\right) / \hbar^{2}\right)^{1 / 2}$. The post-edge absorption background was fitted and subtracted by using a spline function. Then, EXAFS functions were Fourier 
Table 2. Structural data of multielectron excitations.

\begin{tabular}{llll}
\hline Samples & Position $E_{1}(\mathrm{eV})$ & Width $W(\mathrm{eV})$ & Height $H$ \\
\hline${\text { Bulk } \mathrm{CeO}_{2}}$ & 5855 & 5.36 & 0.034 \\
Nanoparticles & 5850 & 8.10 & 0.034 \\
\hline
\end{tabular}

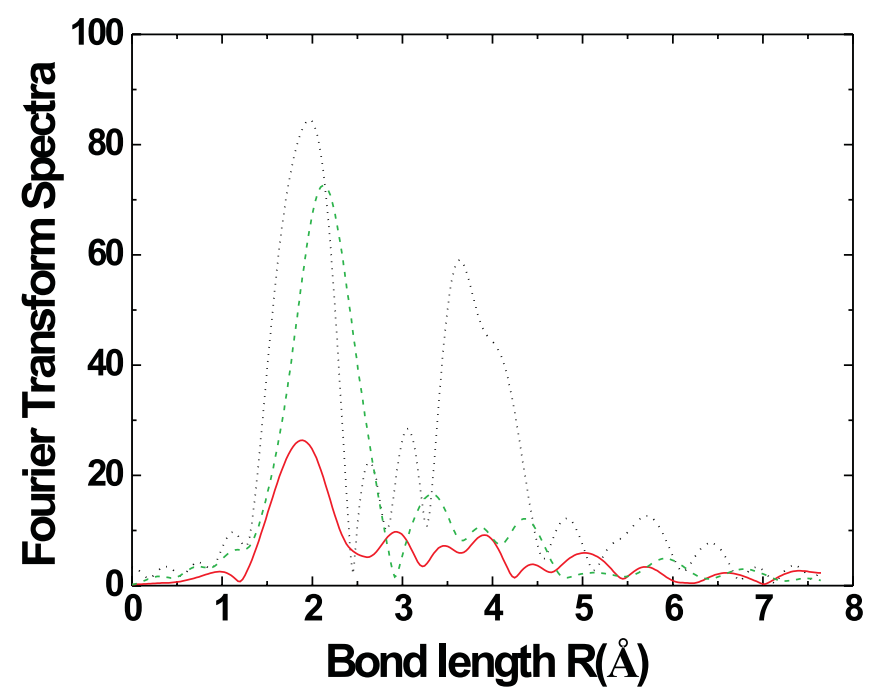

Figure 10. Fourier transform spectra of $\mathrm{Ce}_{I I I}$ absorption in bulk $\mathrm{CeO}_{2}$ (dotted line), AOT-coated cerium oxide nanoparticles (solid line) and hydrous cerium nitrate $\left(\mathrm{Ce}\left(\mathrm{NO}_{3}\right)_{3} \cdot 6 \mathrm{H}_{2} \mathrm{O}\right)$ (dashed line), respectively.

transformed to $R$ space with $k^{2}$-weight in the range of 2.31 to $10.44 \AA^{-1}$. Fourier filters were performed in the range of 1.176 to $2.608 \AA$ A. Seven fitting parameters are allowable. Hanning windows were used in the Fourier transform and filter process. The Fourier-transform spectra and the near-neighbour coordination EXAFS functions are, respectively, shown in figures 10 and 11 .

The common EXAFS formula [31] was used to fit the experimental spectra as follows:

$$
\chi_{j}(k)=\frac{s_{0}^{2} N_{j}}{k R_{j}^{2}} F_{j}(\pi, k) \exp \left(-2 k^{2} \Delta \sigma_{j}^{2}\right) \exp \left(-2 R_{j} / \lambda_{j}\right) \sin \left(2 k R_{j}+\phi_{j}\right) .
$$

Here $N_{j}$ neighbours stand at distance $R_{j}$ away from the absorber. $F_{j}(\pi, k)$ is the backscattering factor, $s_{0}^{2}$ is the reduction factor. $\lambda_{j}$ is the mean free path of the photoelectron, $\phi_{j}$ is the phase shift. $\Delta \sigma_{j}^{2}=\sigma_{u j}^{2}-\sigma_{s j}^{2}$, subscripts $u$ and $s$, respectively, stand for the unknown and reference samples.

Bulk $\mathrm{CeO}_{2}$ was chosen as the reference sample. The back-scattering amplitude $A_{s}(\pi, k)=$ $s_{0}^{2} F(\pi, k) \exp \left(-2 k^{2} \sigma_{s}^{2}\right) \exp \left(-2 R_{s} / \lambda_{s}\right)$ and phase shift $\phi_{s}$ of Ce-O bonds were extracted from the reference spectrum of the $\mathrm{Ce}_{I I I}$ edge in bulk $\mathrm{CeO}_{2}$ with the structural parameters [32] $N=8, R=2.343 \AA$. Due to the transferability of the backscattering amplitude and phase shift, they were substituted for those of the unknown samples in equation (2). One shell is not enough to describe the local atomic structures around Ce because no reasonable result can be obtained. Two shells were used in the fitting procedure. But one of the two $\mathrm{Ce}-\mathrm{O}$ bondlengths was fixed at $2.343 \AA$ because the nanoparticles have the same crystal structures as indicated by the $\mathrm{x}$-ray diffraction pattern shown in figure 2 . Table 3 tabulates the fitting parameters. The fitting curve and the experimental EXAFS function are shown in figure 11. 


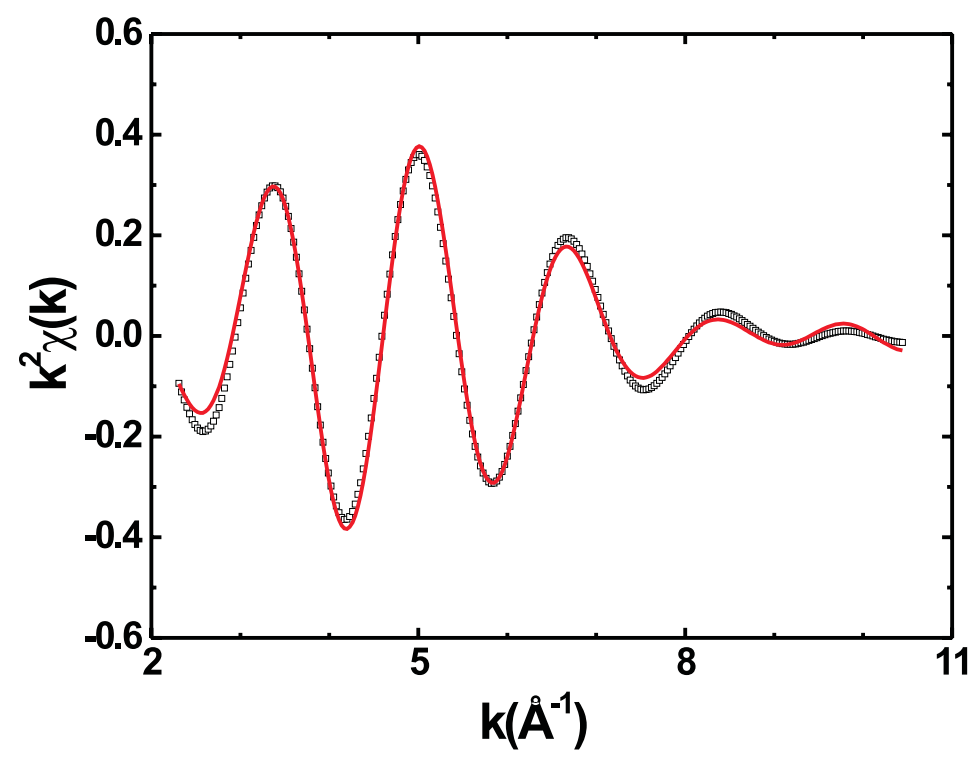

Figure 11. Experimental (points) and fitting (solid line) EXAFS curves of $\mathrm{Ce}-\mathrm{O}$ coordination with $k^{2}$-weight in AOT-coated cerium oxide nanoparticles.

Table 3. Local coordination parameters around Ce in cerium oxide nanoparticles coated with AOT. $N$ is the coordination number, $R$ is the bondlength, $\Delta \sigma^{2}$ is the Debye-Waller factor and $\Delta E_{0}$ is the shift of the energy threshold.

\begin{tabular}{lllll}
\hline Bonds & $N$ & $R(\AA)$ & $\Delta \sigma^{2}\left(\AA^{2}\right)$ & $\Delta E_{0}(\mathrm{eV})$ \\
\hline $\mathrm{Ce}-\mathrm{O}$ & $4.1 \pm 0.3$ & 2.343 & $0.0017 \pm 0.0020$ & $1.0 \pm 1.0$ \\
$\mathrm{Ce}-\mathrm{O}$ & $3.4 \pm 0.4$ & $2.504 \pm 0.020$ & $0.0025 \pm 0.0020$ & $6.8 \pm 1.0$ \\
\hline
\end{tabular}

\section{Results and discussion}

In the EXAFS data analysis, a single-shell fitting cannot give reasonable structural parameters. This implies that the local atomic structures around $\mathrm{Ce}$ in cerium oxide nanoparticles diverge obviously from the single-shell model. We suppose that the nanoparticles consist of two parts, i.e. the shell (surface) part and the core one. The particle size is constrained by the AOT surfactant. Obviously, there is a lattice mismatch in the interface between the surfactant and the nanoparticles. This phenomenon will evidently cause the vacancy and distortion on the interface area. Due to the very small particle size, the ratio of surface to volume should be very large.

From table 3 , the first shell with $\mathrm{Ce}-\mathrm{O}$ bondlength of $2.343 \AA$ corresponds to the core part. The diffraction peaks of cerium oxide nanoparticles as shown in figure 2 are indeed the contribution of the crystalline core parts. Certainly, the core part should have the same crystalline structure as bulk $\mathrm{CeO}_{2}$. The content of the core part can be estimated to be about $51 \%$ based on the coordination number. That is to say, there are about $49 \%$ atoms located at the surface area. If we omit the density difference between the surface part and the core one, the thickness of the surface part can be calculated to be $3 \AA$. The real coordination number around $\mathrm{Ce}$ in the surface area can be calculated to be about 7 according the surface content $49 \%$ and the nominal coordination number 3.4. That is to say, there are about seven oxygen 
atoms around $\mathrm{Ce}$ with the bondlengths of $2.504 \AA$ at the surface of nanoparticles. This is very similar to the local atomic structures around $\mathrm{Ce}$ in crystalline $\mathrm{Ce}_{2} \mathrm{O}_{3}$ [33], in which seven oxygen atoms were separated into 3 subshells with the averaged bondlength of $2.5047 \AA$. This demonstrates that there is a $\mathrm{Ce}_{2} \mathrm{O}_{3}$-like amorphous shell coating on the surface of the core part, while the core part has the $\mathrm{CeO}_{2}$ crystal structure. In crystalline $\mathrm{Ce}_{2} \mathrm{O}_{3}$, the seven oxygen neighbours are separated into three different subshells. But we can only use one shell to describe the local atomic structures of the shell part for the cerium oxide nanoparticles because of the limitation of the number of independent parameters. The shell thickness was estimated to be about $3 \AA$, which only corresponds to one to two bondlengths. It should be easily comprehensible for the nanoparticles to present the amorphous characteristic at such a surface with one to two bondlength thickness. It can also be accepted by using one shell to fit the surface area of the nanoparticles. The EXAFS result is also consistent with the XANES result. In the latter, the $\mathrm{Ce}^{3+}$ and $\mathrm{Ce}^{4+}$ features were found simultaneously in the XANES spectrum of the nanoparticles.

The $\mathrm{x}$-ray diffraction peaks are greatly broadened as shown in figure 2. This can be attributed to the decreasing of the particle size and the increasing of surface to volume ratio. Generally speaking, the shell part of the nanoparticle is easier to distort than the core part. Some lattice mismatch and vacancies will exist in the surface of the nanoparticles or the interface between nanoparticles and surfactant. These distortions probably change the local bondlengths and/or coordination number. With decreasing particle size, the content of surface or interface increases. Therefore, the zone with distortion will increase. In some certain cases, when a single coordination shell is extremely distorted and even split into two subshells, a double-shell model is probably a best choice. Here we consider a two-subshell model to describe the oxygen neighbours surrounding the central $\mathrm{Ce}$ atoms in the cerium oxide nanoparticles. A reasonable fitting curve is obtained as shown in figure 11. We should stress that the multielectron excitation

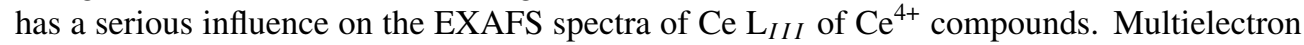
excitation [28] will distort the EXAFS spectrum, which leads a large systematic error and hampers the location of the correct minimum. Without removal of double excitations, a longer bondlength could be obtained. We found the same situation in cerium oxide nanoparticles [34]. It is necessary to subtract the multielectron excitation effect before a reasonable EXAFS data analysis.

The two Ce-O shells have different energy shifts as shown in table 3. Two-shell fitting with consistent energy shifts was also tested. Unless the bondlengths were kept unchanged, the fitting quality was worsened. Debye-Waller factors and coordination numbers were not reasonable. We attribute the energy-shift difference of the two $\mathrm{Ce}-\mathrm{O}$ shells to the coexistence of $\mathrm{Ce}^{3+}$ and $\mathrm{Ce}^{4+}$ components. Each component was described by one $\mathrm{Ce}-\mathrm{O}$ shell with independent energy shift. In fact, because of the two electronic configurations of $4 \mathrm{f}^{0} \mathrm{~L}$ and $4 \mathrm{f}^{1} \mathrm{~L}$ in $\mathrm{Ce}^{4+}$ compounds, two absorption step-edges (corresponding to two energy shifts) were also frequently used in XANES fitting.

In sample preparation, the surfactant AOT formed a 'microreactor' in the solvent. Cerium, which was directly bonded to oxygen, formed cerium oxide nanoparticles within these 'microreactors'. Besides oxygen, other low- $Z$ elements (for example $\mathrm{C}, \mathrm{N}$ and $\mathrm{S}$ ) were also presented in the sample preparation. Carbon and sulphur come from the surfactant AOT. Carbon formed the hydrophobic tail of the surfactant (AOT) soluble only in the organic solvent and remained outside the 'microreactor'. On the other hand, sulphur formed the hydrophilic head (the $-\mathrm{SO}_{3}-$ group) of the surfactant (AOT) and was located inside the 'microreactor'. Sulfur and carbon in surfactants (AOT) are not easy to bond directly to cerium. Nitrogen came only from the starting material $\mathrm{Ce}\left(\mathrm{NO}_{3}\right)_{3} \cdot 6 \mathrm{H}_{2} \mathrm{O}$, in which nitrogen cannot directly bond to cerium. The model with one $\mathrm{Ce}-\mathrm{O}$ shell and another $\mathrm{Ce}-\mathrm{C}$ ( or $\mathrm{Ce}-\mathrm{N}$ or $\mathrm{Ce}-\mathrm{S}$ ) shell was also 
attempted to fit the experimental EXAFS spectrum. The backscattering amplitudes and phase shifts of $\mathrm{Ce}-\mathrm{C}, \mathrm{Ce}-\mathrm{N}, \mathrm{Ce}-\mathrm{O}$ and $\mathrm{Ce}-\mathrm{S}$ bonds were calculated using FEFF [35] software. The following formulae were used to correct the difference between theory and experiment:

$$
\begin{aligned}
& A_{C e-Q}=A_{C e-O}^{E}\left(A_{C e-Q}^{T} / A_{C e-O}^{T}\right) \\
& \phi_{C e-Q}=\phi_{C e-O}^{E}+\left(\phi_{C e-Q}^{T}-\phi_{C e-O}^{T}\right)
\end{aligned}
$$

where $Q$ stands, respectively, for $\mathrm{C}, \mathrm{N}$ and $\mathrm{S}$ elements. $A_{C e-Q}$ and $\phi_{C e-Q}$ are, respectively, the revised amplitude and phase shift. Superscripts $E$ and $T$ denote, respectively, the experimental and calculated values. The experimental ( $\mathrm{Ce}-\mathrm{O}$ bond) or revised $(\mathrm{Ce}-\mathrm{C}, \mathrm{Ce}-\mathrm{N}$ and $\mathrm{Ce}-\mathrm{S}$ bonds) amplitude and phase shift were used in the EXAFS fitting. Obviously, the $\mathrm{Ce}-\mathrm{C}$ and $\mathrm{Ce}-\mathrm{S}$ contributions to the neighbour of cerium can be excluded because of the worse fitting quality and unreasonable fitting parameters. However, with one $\mathrm{Ce}-\mathrm{N}$ shell substituting the $\mathrm{Ce}-\mathrm{O}$ shell of the $\mathrm{Ce}^{3+}$ component, we obtained almost the same structural parameters as listed in table 3 except for a slightly longer $\mathrm{Ce}-\mathrm{N}$ bond $(2.52 \AA)$ than $\mathrm{Ce}-\mathrm{O}$ bond $(2.50 \AA)$. Evidently, nitrogen possesses quite a close electron number to oxygen; the contributions from nitrogen and oxygen neighbours cannot be distinguished in our case using the EXAFS technique. Based on chemical knowledge, we believe that there are no nitrogen neighbours around cerium in these nanoparticles. Probably, the shell part (surface or interface) of nanoparticles does not only consist of cerium and oxygen. For example, cerium may be partially bonded with $\mathrm{OH}^{-}$. This is possible and reasonable either in EXAFS fitting or on chemical knowledge.

Bulk $\mathrm{CeO}_{2}$ belongs to cubic fluorite structure. There are eight $\mathrm{O}$ atoms located in the neighbourhood of Ce with a single $\mathrm{Ce}-\mathrm{O}$ bondlength of $2.343 \AA$ [32]. While in the cerium oxide nanoparticles, due to the decreasing of particle size, the ratio of surface to volume increases. There are about $49 \%$ atoms located at the surface or interface between the nanoparticles and the surfactant AOT. From the structural parameters listed in table 3, we know that the local structure of the surface shell is very like that of $\mathrm{Ce}_{2} \mathrm{O}_{3}$. Comparing with the bulk $\mathrm{CeO}_{2}$ or core part, its bondlengths have been elongated, but the coordination number has decreased slightly. A reasonable explanation is that the AOT surfactant has limited the particle sizes and some oxygen vacancies have been created on the surface area. Further, these oxygen vacancies induce the surface part to a phase transition with $\mathrm{Ce}^{3+}$ oxidation state. Sintering of nano-size $\mathrm{CeO}_{2}$ powders $[36,37]$ led to $\mathrm{CeO}_{2}$ being reduced to $\mathrm{Ce}_{2} \mathrm{O}_{3}$ and oxygen gas being released. Dopants with a valence lower than +4 into $\mathrm{CeO}_{2}$ [38] or argon sputtering and hydrogen exposure to $\mathrm{CeO}_{2}$ [39] caused $\mathrm{Ce}^{3+}$-related defects on the surface of $\mathrm{CeO}_{2}$ and resulted the capping of oxygen vacancies in the surface or a high concentration of $\mathrm{Ce}^{3+}$ in surface and subsurface sites. Those experimental results are similar to our conclusion. Certainly, the morphology and structures of nanoscale materials depend on the methods and procedure of sample preparation. In addition, computer modelling [40] for the $\mathrm{CeO}_{2}$ surface with anion vacancy centres also predicted that generation of vacancies does not seem more favourable than full reduction of (part of) the $\mathrm{CeO}_{2}$ phase to $\mathrm{Ce}_{2} \mathrm{O}_{3}$ for the (111) surface, actually. This prediction is excellently consistent with our experimental results of EXAFS and XANES.

The Debye-Waller factor for the surface area is a little larger than the core part; that is because the larger structural disorder in the former. However, it is still not very large and a symmetrical distribution is enough to describe the local structures of the surface area. In addition, there is no obvious split in the diffraction peaks, which implies a continuous increasing or distortion of $\mathrm{Ce}-\mathrm{O}$ bondlengths from the core to the surface. In fact, EXAFS results give an averaged effect. From this study, we recognize that the surface or interface of the nanoparticle coated with surfactant AOT is quite ordered, although it is an amorphous phase. 


\section{Conclusion}

We studied the local atomic structures of cerium oxide nanoparticles coated with surfactant AOT. From the XANES and EXAFS studies, we conclude that these nanoparticles consist of two parts, i.e. the core part and the surface part. The surface part is amorphous phase with $\mathrm{Ce}_{2} \mathrm{O}_{3}$-like local atomic structures. The $\mathrm{Ce}-\mathrm{O}$ bondlength is greatly elongated in comparison with the bulk one, while the core part is a crystalline phase with almost the same structure as bulk $\mathrm{CeO}_{2}$. The content of the surface part approaches $49 \%$ for the $2-3 \mathrm{~nm}$ cerium oxide nanoparticles with a surface thickness of about $0.3 \mathrm{~nm}$.

\section{Acknowledgments}

Wu Zhonghua is grateful to the Royal Society K C Wong Fellowship and indebted to the financial support by the foundation for returning scholars of the Chinese Academy of Science and the Chinese Ministry of Education. Guo Lin is grateful for the support of the postdoctoral foundation by the Chinese Academy of Sciences. Guo Lin and Li Qianshu thank the Institute of High Education of China for the support of the doctoral programme (No 95968).

\section{References}

[1] Ying J Y 1992 Mater. Lett. 15180

[2] Kubo R 1962 J. Phys. Soc. Japan 17986

[3] Zhou Y C and Rahaman M N 1993 J. Mater. Res. 81680

[4] Hirano M and Kato E 1996 J. Am. Ceram. Soc. 79777

[5] Chebgyun W, Yitai Q, Changsui W, Li Y and Guiwen Z 1996 Mater. Sci. Eng. B 39160

[6] Hirano M and Kato E 1996 J. Mater. Sci. Lett. 151249

[7] Mitsuru H, Yoshikiro K, Masahiro K and Yasuhiko S 1996 J. Mater. Sci. Lett. 151608

[8] Tschoipe A and Ying J Y 1994 Nanostruct. Mater. 4617

[9] Guillou N, Nistor L C, Fuess H and Hahn H 1997 Nanostruct. Mater. 8545

[10] Vallet-Regi M, Conde F and Ragel S V 1997 Mater. Sci. Forum 235-238 291

[11] Toshiyuki M, Kazuyasu F, Ken-ichi M, Gin-ya A, Takao S and Hirotari M 1997 Chem. Mater. 92197

[12] Brust M, Walker M, Bethel D, Schiffrin D J and Whyman R 1994 J. Chem. Soc., Chem. Commun. 801

[13] Beaurepaire E, Kappler J P, Malterre D and Krill G 1988 Europhys. Lett. 5369

[14] Bianconi A, Marceli A, Dexpert H, Karnatak R, Kotani A, Jo T and Petiau J 1987 Phys. Rev. B 35806 Soldatov A V, Ivanchenko T S, Kotani A and Bianconi A 1995 Physica B 208/209 53

[15] Malterre D 1991 Phys. Rev. B 431391

[16] Soldatov A V, Ivanchenko T S, Della Longa S, Kotani A, Iwamoto Y and Bianconi A 1994 Phys. Rev. B 50 5074

[17] Kodre A, Arcon A, Hribar M, Štuhec M, Villain F, Drube W and Tröger L 1995 Physica B 208/209 379

[18] Fonda E, Andreatta D, Colavita P E and Vlaic G 1999 J. Synchron Radiat. 634

[19] Li G G, Bridges F and Brown G 1992 Phys. Rev. Lett. 681609

[20] Mukoyama T and Ito Y 1994 Nucl. Instrum. Methods Phys. Res. B 8726

[21] Chaboy J, Marcelli A and Tyson T A 1994 Phys. Rev. B 4911652

[22] Nachimuthu P, Shih W C, Liu R S, Jang L Y and Chen J M 2000 J. Solid State Chem. 149408

[23] Dexpert H, Karnatak R C, Esteva J-M, Connerade J P, Gasgnier M, Caro P E and Albert L 1987 Phys. Rev. B 361750

[24] Boikov Y A, Claeson T, Erts D, Bridges F and Kvitky Z 1997 Phys. Rev. B 5611312

[25] Capehart T W, Mishra R K, Fuerst C D, Meisner G P, Pinkerton F E and Herbst J F 1997 Phys. Rev. B 5511496

[26] Vandormael D, Grandjean F, Briois V, Middleton D P, Buschow K H J and Long C J 1997 Phys. Rev. B 566100

[27] Paparazzo E, Ingo G M and Zacchetti N 1991 J. Vac. Sci. Technol. A 91416

[28] Benfield R E, Filipponi A, Bowron D T, Newport R J and Gurman S J 1994 J. Phys.: Condens. Matter 68429

[29] D’Angelo P, Di Cicco A, Filipponi A and Pavel N V 1993 Phys. Rev. A 472055

[30] Filipponi A, Tyson T A, Hodgson K O and Mobilio S 1993 Phys. Rev. A 481328 
[31] Sayers D E and Bunker B A 1988 X-ray Absorption: Principle, Applications and Techniques of EXAFS, SEXAFS and XANES ed D C Koningsberger and R Prins (New York: Wiley) ch 6

[32] Whitfield H J, Roman D and Palmer A R 1966 J. Inorg. Nucl. Chem. 282817

[33] Baernighausen H and Schiller G 1985 J. Less-Common Met. 110385

[34] Wu Z H, Guo L, Li H J, Yang Q L, Li Q S and Zhu H S 2000 Mater. Sci. Eng. A 286179

[35] Rehr J J, Zabinsky S I, Ankudinov A and Albers R C 1995 Physica B 208/209 23

[36] Zhou Yanchun and Mohamed N Rahaman 1997 Acta Mater. 453635

[37] Tschopea A and Birringera R 1997 Nanostruct. Mater. 9591

[38] Palmqvist A E C, Wirde M, Gelius U and Muhammed M 1999 Nanostruct. Mater. 11995

[39] Pfau A and Schierbaum K D 1994 Surf. Sci. 32171

[40] Conesa J C 1995 Surf. Sci. 339337 